\title{
Electrochromism in a Gold Cermet
}

\author{
Errol F. I. Roberts
}

Department of Metallurgy and Materials, City of London Polytechnic

The growing demand for visual displays in electronic systems has stimulated research into the phenomenon of electrochromism. This is a general term applied to reversible changes in the visible absorption and reflection spectra of a wide range of solids and liquids subjected to variations in an applied electric field. Such visible changes tend, in electronic terms, to possess rather long switching times (10 to $500 \mathrm{~ms}$ ). Devices based on electrochromism are, however, usually cheap as they can be prepared in large areas, and have economical power consumption characteristics. Consequently a big potential market exists for alpha-numeric displays, on-off indicators, digitalcalculator and watch displays and optical modulation devices (1).

Electrochemical systems exhibiting electrochromism are many and varied. Thus organic redox dyes, such as viologen (2), as well as reversible electroplating (3) have been proposed but electrochemical systems suffer from diffusion problems which, in altering solute ion concentrations, reduce visual contrast and give a loss of colour. Precipitation of coloured species can overcome this but such a deposition reaction is both slow and energy consuming (typically $10 \mathrm{~mJ} / \mathrm{cm}^{2}$ are required during switching). However, a further electrochromic mechanism employing the reversible formation of a tungsten bronze in a reaction of the type:

$$
\mathrm{WO}_{3}+\mathrm{xM}^{+}+\mathrm{xe}^{-} \rightleftharpoons \mathrm{M}_{\mathrm{x}} \mathrm{WO}_{3}
$$

has been suggested and investigated $(4,5,6)$. The tungsten oxide is colourless while the nonstoichiometric compound $\mathrm{M}_{\mathrm{x}} \mathrm{WO}_{3}$ is blue at low $\mathrm{x}$ values. Colouration is induced in a thin film of the transition metal oxide previously deposited on a conducting electrode and immersed in an appropriate electrolyte. It is believed that tungsten oxide forms, with electrons and protons, a compound of formulation $\mathrm{H}_{\mathrm{x}} \mathrm{WO}_{3}$ which is obviously related to $\mathrm{M}_{\mathrm{x}} \mathrm{WO}_{3}$. Indeed, the parent $\mathrm{WO}_{3}$ with a Perovskite structure forms a wide range of such tungsten bronzes without serious distortion of the parent oxide by taking up a range of metallic ions such as lithium, sodium or silver. The colouration process occurs by the simultaneous injection into the oxide film of electrons from a conducting electrode (usually indium-tin oxide) and protons or metal cations from an electrolyte and bleaching occurs by the removal of the injected ions. Such changes are induced by the application of a small voltage. While gels are widely used as electrolytes, solid ionic conductors are of interest for the construction of truly solid-state devices $(7,8)$.

E. K. Sichel, J. I. Gittleman and J. Zelez of RCA Laboratories in Princeton, New Jersey, have recently observed electrochromism in the composite material $\mathrm{Au}-\mathrm{WO}_{3}$ (9). The cermet was prepared in thin film form by the co-sputtering of gold and tungsten oxide onto dielectric substrates with conducting, transparent electrodes. As prepared, such films with a low gold content appear, in transmission, to be transparent and pale blue. Increasing the gold content over 25 atomic per cent causes the films to become metallic and opaque. However, when switched the low gold content films appear pink and those with 25 atomic per cent gold a strong red.

The cermets, as opposed to pure tungsten oxide, appear to possess an auxiliary spectral peak in their optical density curves - at 5900 and $5400 \AA$ respectively for the uncoloured and coloured films. These peaks become less pronounced and broaden with a decrease in gold content but they do not shift in wavelength. A similar occurrence in the $\mathrm{Au}-\mathrm{SiO}_{2}$ system (10) suggested to Sichel and his co-workers the application of the Maxwell-Garnett model. Qualitative agreement with the experimental work appears somewhat disappointing in the light of arbitrary suppression of part of the data. Nevertheless it was observed that, as in the experimental results, colouration resulted in a $400 \AA$ shift of the anomalous absorption towards the blue. The authors suggest that the presence of gold in the cermet prevents colouration of the material to the level encountered in pure $\mathrm{WO}_{3}$. This role of the dispersed gold may be significant if it is able to control spectral features to this extent and goldtransition metal oxide cermets may add to the growing list of novel electro-optic materials.

\section{References}

1 'Non Emissive Electro-optic Displays', edited by A. R. Knetz and F. K. Willisen, Plenum Press, 1976

2 C. J. Shoot et al., Appl. Phys. Lett., 1973, 23, (2), 64-65

3 A. S. Hoffman, 7. Opt..Soc. Am., 1966, 56, (6), 828

4 B. W. Faughnan etal., RCA Rev., 1975, 36, 1977

5 I. F. Chang et al., $\mathcal{F}$. Electrochem. Soc. 1975, 122, 955

6 H. N. Hersh etal., Appl. Phys. Lett., 1976, 28, 95

7 M. Green and D. Richman, Thin Solid Films, 1974, 24, 545

8 M. Green etal., Thin Solid Films, 1966, 38, 89

9 E. K. Sichel etal., Appl. Phys. Lett., 1977, 31, (2), 109-111

10 R. W. Cohen etal., Phys. Rev. B, 1973, 8, 3689 